\title{
Role of Medical Thoracoscopy Guided Pleural Biopsy in Diagnosis of Moderate to Massive Exudative Pleural Effusion
}

Authors

\section{Adesh Kumar ${ }^{1}$ (MD), Aditya Kumar Gautam² (MD), Ashish Kumar Gupta ${ }^{3}$, Somnath Bhattacharya ${ }^{4}$ (MD), Prashant $\operatorname{Yadav}^{5}$ (MD), $\operatorname{Suryakant}^{6}$ (MD)}

${ }^{1}$ Professor, Dept of Respiratory Medicine, U P University of Medical Sciences, Saifai, Etawah, UP

${ }^{2,3}$ Assistant Professor, Department of Respiratory Medicine, U P University of Medical Sciences, Saifai, Etawah, Uttar Pradesh.

${ }^{4}$ Junior Resident, Department of Respiratory Medicine, U P University of Medical Sciences, Saifai, Etawah, Uttar Pradesh.

${ }^{5}$ Senior Resident, Dept of Respiratory Medicine, U P University of Medical Sciences, Saifai, Etawah, UP ${ }^{6}$ Professor, Department of Respiratory Medicine, King George Medical University, Lucknow, Uttar Pradesh Corresponding Author

Dr Aditya K. Gautam

Assistant Professor, Department of Respiratory Medicine, U.P. Medical University Of Medical Sciences, Saifai, Etawah, Uttar Pradesh -206130

Email: adityagautam02@gmail.com, Mobile: 8004959984, 9415787623

\section{ABSTRACT}

Background: Now days medical thoracoscopy has grown interest in establishing the fact of having greater etiological diagnostic yield in exudative moderate to massive pleural effusions. Pleural biopsy under direct vision can be successfully and safely used for etiological diagnosis.

Objective: To evaluate the value of medical thoracoscopic guided parietal pleural biopsy in the etiological diagnosis of exudative moderate to massive pleural effusions at a rural tertiary care centre of north India.

Methods: The study was conducted upon 90 patients with exudative moderate to massive pleural effusion, who required intercostal drainage (ICD) for management. Exudative pleural effusions were also smear negative for acid fast bacilli (AFB) and malignant cytology. All such patients with consent and institutional ethical clearance were submitted for medical thoracoscopic guided pleural biopsy for etiological diagnosis based on histopathology.

Results: Thoracoscopic pleural specimens were diagnostic in 86 patients out of a total 90 patients (95.5\%). Histopathological examination of thoracoscopic specimens revealed, Malignancy-in 31 cases (34.4\%), Inflammatory 28 (31.11\%), Tuberculosis in 25 patients (27.8\%) and Sarcoidosis in two cases (2.22\%). Four patients $(4.4 \%)$ were still inconclusive on histopathology.

Conclusion: Medical thoracoscopic guided pleural biopsy has a good diagnostic yield and safety. Hence it should be put into practice by physicians after proper training.

Keywords: Medical Thoracoscopy, Pleural Biopsy, Pleural Effusion. 


\section{Introduction}

Pleural effusions are a common problem in pulmonary practice and approximately 1.5 million people are believed to develop a pleural effusion in the United States. ${ }^{1}$ The epidemiology of pleural effusions varies depending on the population studied; however, the common causes of exudative pleural effusions (EPEs) worldwide include tubeculosis and malignancy. ${ }^{2}$ Pleural fluid analysis and "blind" pleural biopsy are the initial investigations in patients with EPEs. ${ }^{3}$ Between $20 \%$ and $40 \%$ of patients with EPEs remain undiagnosed despite these investigations. ${ }^{4}$ Larger pleural biopsy specimen taken under direct vision allows greater diagnostic yield above $90 \%$. $^{4}$

The major indication for medical thoracoscopy is evaluation of exudative pleural effusions which remain undiagnosed after pleural fluid analysis, where thoracoscopy is suggested as an alternative to closed pleural biopsy. Medical thoracoscopy has received renewed interest among pulmonary physicians in the recent past because of better instrumentation and simpler sedation protocols. With current techniques, medical thoracoscopy can be done as a day-care procedure under conscious sedation by Pulmonary Physicians. Diagnosis of pleural TB can be achieved in $99 \%$ of patients with thoracoscopy, which is higher than the $51 \%$ yield for closed pleural biopsy. ${ }^{4}$ In the present study, we describe our experience of medical thoracoscopy, diagnostic yield and safety in patients who underwent thoracoscopy for diagnostic purposes.

\section{Material \& Methods}

This was a cross sectional study conducted in male and female patients between 18 to 70 years of age of undiagnosed and moderate to massive exudative pleural effusions. Undiagnosed pleural effusion was defined as failure to achieve a diagnosis by initial pleural fluid analysis including pleural fluid adenosine deaminase (ADA) levels $<70 \mathrm{U} / \mathrm{L}$ and at least three pleural fluid analysis negative for malignant cells. Exudative pleural effusions were confirmed by Light's criteria (as per Lights criteria- (a).Ratio of Pleural fluid protein is to serum Protein $>0.5$ (b). Ratio of Pleural fliud LDH is to Serum LDH >0.6 (c). Level of Pleural fluid $\mathrm{LDH}>2 / 3^{\text {rd }}$ upper normal limit of serum LDH -Any ONE or more) and moderate to massive was assessed by Ultrasonography of thorax and chest X-ray. All patients underwent detailed clinical evaluation with history and clinical examination. Computed tomography (CT) of the chest was performed to assess feasibility of thoracoscopy in certain cases and for lesions in lung parenchyma.

Patients age below 18 and above 70 years, rib crowding with narrow intercostal space and transudative pleural effusion, haemodynamic instability, arrhythmias and intractable cough were excluded from the study . All patients undergoing thoracoscopy were investigated with complete blood count including prothrombin time (PT), activated plasma thrombin time (aPTT) and platelet count to rule out bleeding diathesis. Patients with platelet count less than $50,000 / \mathrm{mm} 3$ and those with PT or aPTT prolonged by more than four seconds above control were also excluded.

Patients were kept fasting for six hours prior to the procedure. Vascular access was achieved with intravenous cannula inserted in the upper limb opposite to the side of thoracoscopy. Patients were positioned in lateral decubitus with diseased side up. Arm on the side of thoracoscopy was positioned above the patient's head. This allowed better access and widens the intercostal spaces. Thoracoscopy was conducted under conscious sedation. Chest wall was draped with sterile cloth after cleaning the skin with $7.5 \%$ povidone iodine. Patients were sedated with intravenous midazolam $(0.5 \mathrm{mg} / \mathrm{kg}$ body weight $)$ and intravenous tramadol $5 \mathrm{mg}$ was given for analgesia prior to the start of procedure. The skin, subcutaneous tissue, intercostal muscle and parietal pleura were anesthetised with $10 \mathrm{~mL} \mathrm{2 \%} \mathrm{lignocaine} \mathrm{to} \mathrm{achieve}$ local anaesthesia. During the procedure intravenous midazolam and tramadol boluses were 
repeated as required to achieve adequate sedation and analgesia. Intravenous pethidine $25 \mathrm{mg}$ was given as bolus to control pain if analgesia could not be achieved with tramadol.

We used single port for visualising and taking pleural biopsy. A $1.5 \mathrm{~cm}$ to $2 \mathrm{~cm}$ long skin incision along the line of intercostal space was given in $5^{\text {th }}$ or $6^{\text {th }}$ intercostal space in mid-axillary line using sterile surgical blade. After blunt dissection of subcutaneous tissue and the intercostal muscles with curved artery forceps, a cannula of $10 \mathrm{~mm}$ diameter with blunt trocar is inserted into the pleural cavity. The trocar was then replaced with semirigid video thoracoscope (Olympus). Pleural fluid was suctioned to enable clear visualisation of entire pleural surface. Thoracoscope was manoeuvred to see visceral, costal, diaphragmatic surface as well as the costophrenic recess. Adhesions were gently lysed using thoracoscope or biopsy forceps to allow visualisation of pleura. After selecting suitable site on parietal pleura for biopsy, biopsy forceps was introduced through working channel of the thoracoscope. Pleura was grasped under vision and biopsy is taken with a shearing movement of the thoracoscope. After the procedure is completed, thoracoscope and the cannula were removed and a 28 to 32 French chest tube was inserted and connected to water-seal drainage bag. Once the lung had expanded and drain output had decreased to less than $50 \mathrm{~mL}$ per 24 hours, chest drain was removed. Demographic characteristics of the patient including the age, gender, clinical diagnosis, pleural fluid analysis, including total and differential count, protein and glucose values, ADA levels, stain for acid-fast bacilli (AFB) and cytology findings and findings of the CT of the chest were recorded.

All data is expressed in percentage and proportions. For the statistical analysis, SPSS software for Windows version 17.0 was used. Continuous data are presented as mean \& standard deviation (SD) Chi-square was used to analyse the collected data where required.

\section{Results}

The mean age of our patients was 47 years. There were 58 male and 32 female cases. There were 51 cases of right sided pleural effusion, 38 cases of left sided pleural effusion and one case of bilateral pleural effusion in our study. Our study showed that thoracoscopic pleural specimens were diagnostic in 86 patients out of a total 90 patients (95.5\%). Four patients $(4.4 \%)$ were still inconclusive on histopathology. Histopathological examination of thoracoscopic specimens revealed: Malignancy- in 31 (34.4\%), Inflammatory 28 (31.11\%), Tuberculosis in $25(27.8 \%)$ and Sarcoidosis in two $(2.22 \%)$ cases.

Out of Malignancy- Poorly-differentiated carcinoma in 13 (41.9\%), Adenocarcinoma in 09 (29.03\%) cases, Squamous cell carcinoma in 03 (9.6\%) cases, Small Round cell tumour / Small cell Carcinoma in 04 (12.9\%) cases \& one case of Mesothelioma and Non Hodgkin's Lymphoma each. Second common finding on histopathology was Inflammatory lesion. Out of 28 cases of inflammatory, there were $03(10.7 \%)$ cases of acute, $15(53.6 \%)$ of chronic and $10(35.71 \%)$ cases of acute on chronic inflammation.

Among 90 patients there were 55 cases $(61.1 \%)$ of Undiagnosed pleural effusions (ADA $<70 \mathrm{U} / \mathrm{L}$ and cytology negative for malignancy) after pleural fluid analysis.. These cases were subjected to medical thoracoscopic guided biopsy and on histology out of 55 case, there were 28 (50.91\%) cases of Malignancy, 13 (23.64\%) of Tubercular, $09(16.36 \%)$ cases of Inflammatory and $02(4 \%)$ cases of Sarcoidosis and $03(5.45 \%)$ cases were still inconclusive. Our overall diagnostic yield was $94.5 \%$ (52/55 patients).

Though complications were few yet analysis showed, bleeding in three cases, surgical emphysema in two cases, poor wound healing in two cases and nonhealing pleurocutaneous fistula in one case. 
Table-1: Etiological Profile of Exudative Pleural Effusion Based On Pleural Histopathology

\begin{tabular}{|l|l|c|}
\hline S/N & Histopathology & $\begin{array}{c}\mathbf{N}=\mathbf{9 0} \\
\mathbf{( 1 0 0 \% )}\end{array}$ \\
\hline $\mathbf{1 .}$ & Malignanacy & $\mathbf{3 1 ( 3 4 . 4 4 \% )}$ \\
& Poorly-differentiated carcinoma & $13(41.9 \%)$ \\
& Adenocarcinoma & $09(29.03 \%)$ \\
& Squamous cell carcinoma & $03(9.6 \%)$ \\
& Small cell carcinoma & $04(12.90)$ \\
& Mesothelioma & $01(3.22 \%)$ \\
& Nonhodgkins lymphoma & $01(3.22 \%)$ \\
\hline $\mathbf{2 .}$ & Tuberculosis & $\mathbf{2 5}(\mathbf{2 7 . 7 7 \%})$ \\
\hline $\mathbf{3 .}$ & Inflammation & $\mathbf{2 8 ( 3 1 . 1 1 \% )}$ \\
\hline $\mathbf{4 .}$ & Sarcoidosis & $\mathbf{0 2}(\mathbf{2 . 2 2 \% )}$ \\
\hline $\mathbf{5 .}$ & Nonconclusive & $\mathbf{0 4}(\mathbf{4 . 4 4 \% )}$ \\
\hline
\end{tabular}

Table-2: Etiological Profile of Undiagnosed Exudative Pleural Effusion after thoracoscopic pleural biopsy and Histopathology

\begin{tabular}{|l|l|c|}
\hline S/N & Histopathology & $\begin{array}{c}\mathbf{N}=\mathbf{5 5} \\
(\mathbf{1 0 0 \%})\end{array}$ \\
\hline $\mathbf{1 .}$ & Malignancy & \\
& Poorly-differentiated & $\mathbf{2 8 ( 5 0 . 9 1 \% )}$ \\
& carcinoma & $10(35.71 \%)$ \\
& Adenocarcinoma & $9(32.14 \%)$ \\
& Small cell carcinoma & $3(10.71 \%)$ \\
& Squamous cell carcinoma & $4(14.28 \%)$ \\
& Mesothelioma & $1(3.57 \%)$. \\
& Nonhodgkins lymphoma & $1(3.57 \%)$. \\
\hline $\mathbf{2 .}$ & Tuberculosis & $\mathbf{1 3}(\mathbf{2 4 \%})$ \\
\hline $\mathbf{3 .}$ & Inflammation & $\mathbf{0 9}(\mathbf{1 6 \%})$ \\
\hline $\mathbf{4 .}$ & Sarcoidosis & $\mathbf{0 2}(\mathbf{4 \%})$ \\
\hline $\mathbf{5 .}$ & Nonconclusive & $\mathbf{0 3}(\mathbf{5 \%})$ \\
\hline
\end{tabular}

\section{Discussion}

In this study, we have presented the data of 90 consecutive patients who underwent thoracoscopy for the diagnosis of exudative pleural effusions. We included patients with undiagnosed pleural effusions for thoracoscopy in whom initial diagnostic work-up with pleural fluid analysis including pleural fluid ADA and three pleural fluid cytologies were inconclusive. The Overall yield of thoracoscopic pleural biopsy for exudative effusion was $95.5 \%$ (86/90) patients and for undiagnosed effusion was $94.5 \% \quad(52 / 55$ patients). Similar experience with medical thoracoscopy has been described from other centers. Kendall et al reported yield of thoracoscopic pleural biopsy to be $83 \%$ in their study which included 48 patients. ${ }^{5}$ Tscheikuna et al described their experience from Thailand $(\mathrm{n}=86)$ and thoracoscopy was diagnostic in $95 \%$ of 34 patients. $^{6} \mathrm{Ng}$ et al could achieve diagnosis with thoracoscopic pleural biopsy in $45.5 \%$ $(10 / 22)$ patients with undiagnosed pleural effusions. $^{7}$ In a majority of patients in our study, thoracoscopic pleural biopsy yielded diagnosis of pleural malignancy of yield $46.67 \%$ and Tuberculosis $26.7 \%$. Similar observations were made by Tscheikuna et al who found pleural malignancy in $45 \%$ of patients with undiagnosed pleural effusions undergoing thoracoscopy. ${ }^{6} \mathrm{Ng}$ et al found that $45.5 \%$ of patients with undiagnosed pleural effusions had pleural malignancy. ${ }^{7}$ A significant proportion of patients, $45.7 \%(16 / 35)$ with un-diagnosed pleural effusion had pleural malignancy in VK Mootha et al study from India. ${ }^{8}$ Pleural metastasis is the more common cause of malignant pleural effusions than mesothelioma. We could diagnose only one case of Mesothelioma whereas 30 of the 31 cases were due to pleural metastasis. Among the patients with metastatic pleural effusion from lung cancer, Poorly differentiated lung carcinoma was the most common diagnosis with 13 patients followed by Adenocarcinoma. Among those with malignant pleural effusion from primary lung cancer, Nine out of 31 patients had Adenocarcinoma, Three had Squamous cell lung cancer, four had Small cell Carcinoma and one had Lymphoma. Probably with help of Immunohistochemistry (IHC) we could classify poorly differentiated Carcinoma into further subtypes giving these findings in concordance with the findings of others studies showing Adenocarcinoma as the most common diagnosis worldwide., ${ }^{\mathbf{9} 10} 25$ out of 90 (27.8\%) patients in whom we performed thoracoscopy had pleural TB on pleural biopsy and among undiagnosed effusions the yield was 13 out of 55 patients $(23.64 \%$ only) and for malignant in undiagnosed effusion was $50.91 \% \quad(28 / 55$ patients).

This is in similarity to the findings of Kendall et al who did not find any case of TB in their study of 48 patients undergoing thoracoscopy for undiagnosed pleural effusions. ${ }^{\mathbf{5}}$ This is probably 
due to low prevalence of TB in the West but a rising trend of lung malignancy in our country. Our study had a overall complications in $11(12.2 \%)$ cases of which we had- bleeding in three $(3.33 \%)$ cases, Non healing pleurocutaneous fistula in one $(1.11 \%)$ case and Surgical emphysema in two $(2.22 \%)$ cases and Death in three $(1.33 \%)$ cases and poor wound healing two (2.22\% cases). A variety of complications associated with thoracoscopy have been described in the literature such as subcutaneous emphysema $(0.6 \%-4.9 \%)$, air leak $(0.5 \%-8.1 \%)$, empyema $(0.5 \%-2.7 \%)$, haemorrhage $(0.3 \%-0.4 \%)$, shock $(0.2 \%)$, chest wall seeding by malignancy $(0.5 \%$ $4.0 \%)^{6,11-15}$

Thus thoracoscopic pleural biopsy is considered gold standard in diagnosis of malignant pleural effusion and tubercular pleural effusion. Diagnostic yield of thoracoscopic pleural biopsy can be as high as $95 \%$ in malignant pleural effusions and $99 \%$ in TB pleural effusions which is far superior to that of pleural fluid analysis and closed pleural biopsy. ${ }^{12}$ These findings along with results of our study and similar studies mentioned above suggest that thoracoscopic pleural biopsy should be considered in all patients with pleural effusions who remain undiagnosed after initial pleural fluid analysis.

\section{Conclusion}

Medical thoracoscopy in our study had a high Overall Diagnostic yield - 95.5\% of which Malignancy-34.4\% , Inflammatory-31.11\% and Tuberculosis - 27.8\%. In Undiagnosed pleural effusion (ADA $<70 \mathrm{U} / \mathrm{L}$, cytology negative for malignancy) Over all diagnostic yield was $94.5 \%$ with Malignancy-50.91\% and TB-23.64\% from our study. Malignancy being the leading cause. Pleural metastasis (30/31cases) due to Poorly differentiated Lung Carcinoma being the leading cause (which needed IHC for subtypes) followed by Adenocarcinoma being more common than Mesothelioma (1/31 cases) in our study. It is relatively simple, easy to use and safe procedure with minimal complications after proper training.
Therefore should be considered in patients with undiagnosed pleural effusion where tuberculosis and malignancy are clinical possibilities and initial pleural fluid analysis is inconclusive.

\section{Article presented at Confrences}

Organization: Bronchocon 2016

Place: Srinagar

Date: April 2016

Organization: NAPCON 2016

Place: Mumbai

Date: Nov. 2016

Sources of support - nil

\section{References}

1. Light RW . Pleural effusions. Med Clin North Am . 2011 ;95 ( 6 ): 1055 - 1070 .

2. Porcel JM , Vives M . Etiology and pleural fluid characteristics of large and massive effusions. Chest .2003; 124 ( 3 ): 978 983 .

3. Sahn SA. The differential diagnosis of pleural effusions . West J Med . $1982 ; 137$ ( 2 ): $99-108$

4. Loddenkemper R, Grosser H, Gabler A, Mai J, Presseuler H, Brandt HJ. Prospective evaluation of biopsy methods in diagnosis of malignant pleural effusions: intra patient comparision between pleural fluid cytology, blind needle biopsy and thoracoscopy. Am Rev Respir Dis 1983;127:114.

5. Kendall SW, Bryan AJ, Large SR, Wells FC. Pleural effusions: is thoracoscopy a reliable investigation? A retrospective review. Respir Med 1992;86:437-40.

6. Tscheikuna J, Silairatana S, Sangkeaw S, Nana A. Outcome of medical thoracoscopy. J Med Assoc Thai 2009; 92 (Suppl. 2):S19-S23.

7. $\mathrm{Ng} \mathrm{TH}$, How SH, Kuan YC, Hasmah H, Norra H, Fauzi AR. Medical thoracoscopy: Pahang experience. Med J Malaysia 2008;63:298-301. 
8. V.K. Mootha, R. Agarwal, N. Singh, A.N. Aggarwal, D. Gupta and S.K. Jindal. Medical Thoracoscopy for Undiagnosed Pleural Effusions:Experience from a Tertiary Care Hospital in North India. The Indian Journal of Chest Diseases \& Allied Sciences 2011; Vol.53:21-24.

9. Johnston WW. The malignant pleural effusion: a review of cytopathologic diagnoses of 584 specimens from 472 consecutive patients. Cancer 1985;56:9059.

10. Chernow B, Sahn SA. Carcinomatous involvement of the pleura: an analysis of 96 patients. Am J Med 1977;63:695-702.

11. Casal RF, Eapen GA, Morice RC, Jimenez CA. Medical thoracoscopy. Curr Opin Pulm Med 2009;15:313-20.

12. Boutin C, Viallat JR, Cargnino P, Farisse P. Thoracoscopy in malignant pleural effusions. Am Rev Respir Dis 1981;124:588-92.

13. Viskum K, Enk B. Complications of thoracoscopy.Poumon Coeur1981;37:25-8.

14. Menzies R, Charbonneau M. Thoracoscopy for the diagnosis of pleural disease. Ann Intern Med1991;114:271-6.

15. de Campos JR, Vargas FS, de Campos Werebe E, CardosoP, Teixeira LR, Jatene FB, et al. Thoracoscopy talc poudrage: a 15-year experience. Chest 2001;119:801-6.

16. Blanc FX, Atassi K, Bignon J, Housset B. Diagnostic value of medical thoracoscopy in pleural disease: A 6 year retrospective study. Chest 2002;121:1677-83. 